\title{
Effects of etomidate on bispectral index scale and spectral entropy during induction of anesthesia by means of the raw electroencephalographic and electromyographic characteristics
}

\author{
Hyun-Mok Kim, Sang-Wook Shin, Ji-Young Yoon, Hyeon-Jeong Lee, Kyung-Hoon Kim, and \\ Seong-Wan Baik
}

Department of Anesthesia and Pain Medicine, Pusan National University School of Medicine, Yangsan, Korea

Background: Etomidate frequently induces myoclonus, so it may affect electromyographics (EMG). And EMG commonly has an effect on the bispectral index scale (BIS) and spectral entropy. This study was performed to compare the effect of etomidate on BIS, response entropy (RE) and state entropy (SE) during induction of anesthesia. Methods: Fifty patients (ASA I or II) scheduled for elective surgery were included in this study. Anesthesia was induced with etomidate $(0.3 \mathrm{mg} / \mathrm{kg})$ and rocuronium $(0.6 \mathrm{mg} / \mathrm{kg})$. Patients also inhaled 4 vol\% sevoflurane and $100 \%$ oxygen and, then intubated. BIS, RE, SE and Modified Observer's Assessment of Alertness/Sedation Scale (MOAA/S) were measured 4 times (before injection of etomidate [T0], at loss of eyelash reflex [T1], 90 seconds after rocuronium injection [T2], and after intubation [T3]). We also checked whether myoclonus occurred.

Results: Baseline values (T0) were $93.1 \pm 4.7$ for BIS, $95.8 \pm 3.7$ for RE and, $87.3 \pm 3.5$ for SE. In comparison with T0, there were significantly differences in BIS (50.2 \pm 16.3$)$, RE (76.8 \pm 18.5$)$ and SE (66.3 \pm 17.4$)$ at T1 (all P $<0.05)$. There were no significant differences at T2 and T3. Thirty one patients had myoclonus. At the occurrence of myoclonus, RE and SE values significantly increased but not BIS $(\mathrm{P}<0.05)$.

Conclusions: In patients with myoclonus, at the loss of consciousness, spectral entropy did not decrease where as BIS did, suggesting that BIS may evaluate hypnotic levels better than spectral entropy during induction of anesthesia with etomidate. (Korean J Anesthesiol 2012; 62: 230-233)

Key Words: Bispectral index scale, Electromyography, Entropy, Etomidate, Myoclonus.

Received: April 1, 2011. Revised: 1st, May 20, 2011; 2nd, June 13, 2011. Accepted: July 16, 2011.

Corresponding author: Sang-Wook Shin, M.D., Department of Anesthesia and Pain Medicine and Medical Research Institute, Pusan National University School of Medicine, Beomeo-ri, Mulgeum-eup, Yangsan 626-770, Korea. Tel: 82-55-360-2129, Fax: 82-55-360-2149, E-mail: shinsw@ pusan.ac.kr

(c) This is an open-access article distributed under the terms of the Creative Commons Attribution Non-Commercial License (http:// creativecommons.org/licenses/by-nc/3.0/), which permits unrestricted non-commercial use, distribution, and reproduction in any medium, provided the original work is properly cited. 


\section{Introduction}

The bispectral index scale (BIS) and spectral entropy are commonly used to evaluate the depth of hypnosis. They are all electroencephalographic (EEG)-derived indices. BIS is composed of EEG parameters that integrates several disparate descriptors of the EEG into a single variable and is the most commonly used scale to judge and control hypnotic levels during general anesthesia and sedation [1]. On the other hand, spectral entropy reflects raw EEG and frontal electromyography (FEMG) data, resulting in two values, namely response entropy (RE) and state entropy (SE). SE, which is calculated over frequencies ranging from 0.8 to $37 \mathrm{~Hz}$, is the entropy of the EEG signal reflecting the patient's cortical activity. RE includes additional higher frequencies up to $47 \mathrm{~Hz}$, reflecting both EEG and FEMG activity.

Both BIS and spectral entropy may be influenced by several factors. Drugs and surgical stimulation can change values of BIS and spectral entropy [2,3]. Some reports showed that electromyography (EMG) activity has an effect on BIS and spectral entropy [4]. BIS and spectral entropy is calculated over frequencies that include EEG and EMG, so EMG activity may change values of BIS and spectral entropy.

Etomidate maintains cardiovascular stability during induction of general anesthesia, so it can be used relatively safely in patients with compromised myocardial contractility. But, it often causes myoclonus to affect EMG $[5,6]$. Accordingly, author hypothesized that EMG activity after etomidate injection would affect interpretation of hypnotic levels as determined by BIS and spectral entropy. This study was performed to compare the effect of etomidate on BIS, RE and SE during induction of anesthesia.

\section{Materials and Methods}

After approval from the regional hospital ethics committee and informed consent, authors studied 50 patients, American Society of Anesthesiologists class I-II, 20-69 years of age, undergoing elective surgery. Exclusion criteria included disease or injury affecting the central nervous system, recent use of psychoactive or analgesic medication, neurological disorders, alcohol, or drug abuse, and body weight below $70 \%$ or above $130 \%$ of the patient's ideal body weight.

Patients arrived at the operating room after an overnight fast. Glycopyrrolate ( $0.2 \mathrm{mg}$ ) was given intramuscularly 1 hour before surgery as premedication. They were monitored with electrocardiography, pulse oximetry, end tidal carbon dioxide and non invasive blood pressure measurements. BIS was monitored with the A-3000 BIS monitor (Aspect Medicine system Inc., Norwood, USA). RE and SE were monitored with a Datex-Ohmeda Aestiva/5 Entropy Module (E-Entropy ${ }^{\mathrm{TM}}$, GE
Table 1. Modified Observer's Alertness/Sedation scale (MOAA/S)

\begin{tabular}{cl}
\hline Score & \multicolumn{1}{c}{ Description } \\
\hline 5 & Responds readily to name spoken in normal tone \\
4 & Lethargic response to name spoken in normal tone \\
3 & Responds only after name is called loudly and/or repeatedly \\
2 & Responds only after mild prodding or shaking \\
1 & Responds only after painful trapezius squeeze \\
0 & No response after painful trapezius squeeze \\
\hline
\end{tabular}

Healthcare, Helsinki, Finland). Mean arterial pressure (MAP), heart rate (HR), Modified Observer's Assessment of Alertness/ Sedation scale (MOAA/S) (Table 1), BIS, RE and SE were recorded every $5 \mathrm{~min}$.

Anesthesia was induced with etomidate $(0.3 \mathrm{mg} / \mathrm{kg})$, then rocuronium $(0.6 \mathrm{mg} / \mathrm{kg})$ was injected in patients after loss of eyelash reflex and patients inhaled $100 \%$ oxygen. Ninety seconds after rocuronium injection, the patients inhaled 4 vol\% sevoflurane and $100 \%$ oxygen, and then they were intubated.

BIS, RE, SE and MOAA/S were measured 4 times (before injecting etomidate [T0], after the loss of eyelash reflex [T1], 90 seconds after rocuronium injection [T2], and after intubation [T3]). In addition, authors checked whether myoclonus occurred during the induction period of anesthesia.

Data were expressed as means \pm standard deviation (SD) and analyzed using a two-way mixed-designed analysis of variance (ANOVA) and Tukey's post hoc test for comparisons. Differences in BIS, RE, SE, MAP, and HR between group with myoclonus and group without myclonus were analyzed with two-way analysis of variance followed by paired t-test. The number of patients is indicated by $n$. Probability values of (P) $<0.05$ were considered statistically significant. All statistical analyses were performed using the Statistical Package for Social Sciences (SPSS) version 12.0 (SPSS Inc, Chicago, IL, USA).

\section{Results}

A total of 50 patients were included. Mean age, weight, height, MAP and HR were $37.4 \pm 11.4$ yrs (range 19-61), $64.0 \pm$ $12.4 \mathrm{~kg}$ (range 47.0-110.0), $165.6 \pm 9.0 \mathrm{~cm}$ (range 146.0-186.0), $98.0 \pm 13.9 \mathrm{mmHg}$ (range $73-132$ ) and $76.8 \pm 18.1$ beats $/ \mathrm{min}$ (range 50-119), respectively. Twenty-one patients (42\%) were males and 29 were females (58\%).

BIS, RE and SE values at baseline (T0) and the values after injection of etomidate (T1, T2, T3) are summarized in Table 2. Compared with T0, the T1 values for BIS 50.2 \pm 16.3 , RE 76.8 \pm 18.5 , and SE $66.3 \pm 17.4$ decreased $(\mathrm{P}<0.05)$. There were no significant differences among BIS, RE and SE at T2 and T3. All MOAA/S scale at T1, T2, and T3 were below 2.

Among a total 50 patients, 31 patients had myoclonus. There were no statistically significant differences in the gender, 
Table 2. BIS, RE and SE Values at Each Point after Etomidate Injection

\begin{tabular}{clll}
\hline & \multicolumn{1}{c}{ BIS } & \multicolumn{1}{c}{ RE } & \multicolumn{1}{c}{ SE } \\
\hline T0 & $93.1 \pm 4.7$ & $95.8 \pm 3.7$ & $87.3 \pm 3.5$ \\
T1 & $50.2 \pm 16.3^{*}+\dagger$ & $76.8 \pm 18.5$ & $66.3 \pm 17.4^{*}$ \\
T2 & $40.1 \pm 10.2$ & $42.4 \pm 16.1$ & $37.3 \pm 15.9$ \\
T3 & $40.8 \pm 11.3$ & $38.2 \pm 15.9$ & $34.5 \pm 15.0$ \\
\hline
\end{tabular}

BIS: bispectral index scale, RE: response entropy, SE: state entropy. T0: baseline, T1: when loss of eyelash reflex, T2: 90 seconds after rocuronium injection, T3: after intubation. ${ }^{*} \mathrm{P}<0.05 \mathrm{vs} \mathrm{RE},{ }^{\dagger} \mathrm{P}<0.05$ vs SE Values are means \pm SD.

Table 3. Patient Characteristics According to Occurrence of Myoclonus

\begin{tabular}{lccc}
\hline & $\begin{array}{c}\text { With myoclonus } \\
(\mathrm{n}=31)\end{array}$ & $\begin{array}{c}\text { Without myoclonus } \\
(\mathrm{n}=19)\end{array}$ & P value \\
& & & 0.079 \\
Sex & $16(52 \%)$ & $5(26 \%)$ & \\
$\quad$ Male (\%) & $15(48 \%)$ & $14(74 \%)$ & \\
$\quad$ Female (\%) & $34.7 \pm 10.8$ & $42.0 \pm 11.3$ & 0.027 \\
Age (yr) & $66.5 \pm 13.9$ & $59.8 \pm 8.1$ & 0.059 \\
Weight (kg) & $166.8 \pm 9.1$ & $163.8 \pm 8.7$ & 0.270 \\
Height (cm) & $95.3 \pm 18.9$ & $101.3 \pm 11.5$ & 0.222 \\
MAP (mmHg) & mats & $80.7 \pm 14.7$ & 0.531 \\
HR (beats/min) & $77.8 \pm 16.9$ & &
\end{tabular}

Values are means \pm SD. MAP: mean arterial pressure, HR: heart rate.

weight, height, MAP and HR between patients with or without myoclonus (Table 3). On the other hand, younger age was significantly associated with myoclonus (Table 3). RE and SE values in the myoclonus group were significantly higher than those without myoclonus at T1, but BIS was similar, whether myoclonus occurred or not (Table 4).

\section{Discussion}

The main finding of this study was that BIS was lower than RE and SE at the point of immediate loss of consciousness after etomidate injection. BIS score was under 60 regardless of myoclonus at loss of eyelash reflex, whereas RE and SE were over 60. Moreover, RE and SE in patients with myoclonus were higher than those of patients without myoclonus, but BIS was not different.

Etomidate is often used for inducing anesthesia in patients who have limited hemodynamic reserve. But, it has several disadvantages like dose-dependent adrenocortical suppression, possible inhibition of platelet function, and involuntary skeletal muscle contractions (myoclonus). Involuntary skeletal movement characterized as myoclonus occurred more than $50 \%$ of patients receiving etomidate [7], and when myoclonus occurs, EMG readings may change. EMG activity may influence BIS and spectral entropy [4], by interrupting the interpretation of hypnotic levels after etomidate injection.

According to this study, RE and SE did not decrease the same
Table 4. BIS, RE and SE Values with Myoclonus at T1

\begin{tabular}{lccc}
\hline & $\begin{array}{c}\text { With myoclonus } \\
(\mathrm{n}=31)\end{array}$ & $\begin{array}{c}\text { Without myoclonus } \\
(\mathrm{n}=19)\end{array}$ & P value \\
\hline $\mathrm{BIS}$ & $46.7 \pm 10.1$ & $47.2 \pm 11.0$ & 0.862 \\
$\mathrm{RE}$ & $79.9 \pm 15.6$ & $62.4 \pm 21.8$ & 0.005 \\
$\mathrm{SE}$ & $67.9 \pm 13.6$ & $55.8 \pm 20.4$ & 0.029 \\
\hline
\end{tabular}

Values are means \pm SD. BIS: bispectral index scale, RE: response entropy, SE: state entropy.

as BIS at loss of consciousness during induction of anesthesia with etomidate. Values were $50.2 \pm 16.3$ (BIS), $76.8 \pm 18.5$ (RE), $66.3 \pm 17.4$ (SE) at T1. Among them, RE and SE values were over 60 at $\mathrm{T} 1$, which are regarded as inappropriate hypnotic levels for intubation. It is thought that RE and SE values of patients with myoclonus increased their mean values, compared with BIS. The appropriate hypnotic level for intubation is known to be below 60 for BIS or spectral entropy, in general [8].

Spectral entropy consists of RE and SE. RE is calculated over frequencies in the range $0.8-47 \mathrm{~Hz}$ including EMG, where frequencies in the range $32-47$ reflect EMG; therefore, $\mathrm{RE}$ is dominantly influenced by EMG. SE, which is calculated over frequencies ranging from 0.8 to $37 \mathrm{~Hz}$, is the entropy of the EEG signal. In this frequency range, most EMG activity is eliminated and SE commonly is not affected. But sometimes EMG can affect SE, because the power spectrum of EMG overlaps that of EEG, and strong EMG impact may change the spectrum at 20 $\mathrm{Hz}[9]$.

The BIS calculates three sub parameters including the burst suppression ratio, the beta ratio and SynchFastSlow. The burst suppression ratio is the proportion of the suppressed EEG (isoelectric) in an epoch, the beta ratio is the log ratio of the power in two empirically derived frequency bands (highand medium-frequency ranges), and SynchFastSlow is the relative bispectral power in the $40-47 \mathrm{~Hz}$ frequency band [10]. The bispectral analysis examines the relationship between the sinusoids at two primary frequencies, f1 and $\mathrm{f} 2$, and a modulation component of the frequency $\mathrm{f} 1+\mathrm{f} 2$.

Bruhn et al. [4] reported two cases of BIS increase by EMG activity in anesthetized patients. Another case where BIS increased by myoclonus during etomidate injection was also reported [11]. It is supposed that calculation of BIS requires inclusion of frequencies above $40 \mathrm{~Hz}$, and approaching frequencies generated by muscle activity on EMG, such as SynchFastSlow.

In some studies, noxious pain like tracheal intubation increases spectral entropy, especially RE rather than BIS [12,13], since noxious pain is relevant to increases EMG activity [14]. By comparison, we investigated BIS, RE and SE values during induction of anesthesia, and not in anesthetized patients. In this case, BIS decreased significantly rather than entropy when the 
level of consciousness is decreased. When myoclonus occurred, differences between BIS and spectral entropy were larger and RE had the largest difference among BIS, SE and RE. It seems that BIS may be interfered by EMG less than entropy and mainly influenced by EEG. Further studies are needed to determine the mechanism that etomidate influences BIS and spectral entropy.

In the present study, the occurrence of myoclonus after etomidate injection was $62 \%$ in patients, and it was not significantly related to sex, weight, height and vital signs, whereas the mean age of patients who expressed myoclonus was younger than those who did not. The authors propose that younger patients may have more muscle mass, which might affect to the occurrence of myoclonus. It is necessary for additional studies investigating the association between age and myoclonus.

In this study, there was no significant difference between BIS, RE and SE after injection of the muscle relaxant. Muscle relaxants suppress muscle movement, so it may affect data like EMG activity. Several studies reported that muscle relaxants decrease BIS, RE and SE [15,16]. As a result, hypnotic levels may be misinterpreted by EMG activity $[9,17]$. The mechanisms of etomidate induced myoclonus are not clarified yet. Myoclonus has features like tonic-clonic seizures, as muscle movements generally increase [6]. When myoclonus occurred, RE and SE values were higher than those without myoclonus at T1, but BIS was rarely influenced by existence of myoclonus. It is presumed that RE and SE were strongly influenced by EMG activity.

Finally, when myoclonus occurred, RE and SE did not decrease similarly to BIS at the level of loss of consciousness during induction of anesthesia with etomidate. Therefore, BIS may reflect hypnotic levels better than spectral entropy in patients with myoclonus during induction of anesthesia with etomidate.

\section{References}

1. Liu J, Singh H, White PF. Electroencephalogram bispectral analysis predicts the depth of midazolam-induced sedation. Anesthesiology 1996; 84: 64-9.

2. Hans P, Dewandre PY, Brichant JF, Bonhomme V. Comparative effects of ketamine on Bispectral Index and spectral entropy of the electroencephalogram under sevoflurane anaesthesia. Br J Anaesth 2005; 94: 336-40.
3. Choi SH, Kim CS, Kim JH, Kim BS, Kim EM, Min KT. A single dose of esmolol blunts the increase in bispectral index to tracheal intubation during sevoflurane but not desflurane anesthesia. J Neurosurg Anesthesiol 2009; 21: 214-7.

4. Bruhn J, Bouillon TW, Shafer SL. Electromyographic activity falsely elevates the bispectral index. Anesthesiology 2000; 92: 1485-7.

5. Gultop F, Akkaya T, Bedirli N, Gumus H. Lidocaine pretreatment reduces the frequency and severity of myoclonus induced by etomidate. J Anesth 2010; 24: 300-2.

6. Choi JM, Choi IC, Jeong YB, Kim TH, Hahm KD. Pretreatment of rocuronium reduces the frequency and severity of etomidateinduced myoclonus. J Clin Anesth 2008; 20: 601-4.

7. Do SH, Han SH, Park SH, Kim JH, Hwang JY, Son IS, et al. The effect of injection rate on etomidate-induced myoclonus. Korean J Anesthesiol 2008; 55: 305-7.

8. Bein B. Entropy. Best Pract Res Clin Anaesthesiol 2006; 20: 101-9.

9. Aho AJ, Yli-Hankala A, Lyytikäinen LP, Jäntti V. Facial muscle activity, response entropy, and state entropy indices during noxious stimuli in propofol-nitrous oxide or propofol-nitrous oxide-remifentanil anaesthesia without neuromuscular block. Br J Anaesth 2009; 102: 227-33.

10. Tempe DK, Satyanarayana L. Is there any alternative to the Bispectral Index Monitor? Br J Anaesth 2004; 92: 1-3.

11. Lim TA, Lim KY. BIS during etomidate-induced myoclonus. Anaesthesia 2006; 61: 410-1.

12. Wheeler P, Hoffman WE, Baughman VL, Koenig H. Response entropy increases during painful stimulation. J Neurosurg Anesthesiol 2005; 17: 86-90.

13. Takamatsu I, Ozaki M, Kazama T. Entropy indices vs the bispectral index for estimating nociception during sevoflurane anaesthesia. Br J Anaesth 2006; 96: 620-6.

14. Kawaguchi M, Takamatsu I, Masui K, Kazama T. Effect of landiolol on bispectral index and spectral entropy responses to tracheal intubation during propofol anaesthesia. Br J Anaesth 2008; 101: 273-8.

15. Kim BS, Lee SI, Choi SU, Shin HW, Lim HJ, Lee HW, et al. Effect of muscle relaxant on entropy during propofol anesthesia. Korean J Anesthesiol 2009; 56: 381-6.

16. Liu N, Chazot T, Huybrechts I, Law-Koune JD, Barvais L, Fischler M. The influence of a muscle relaxant bolus on bispectral and datexohmeda entropy values during propofol-remifentanil induced loss of consciousness. Anesth Analg 2005; 101: 1713-8.

17. Vivien B, Di Maria S, Ouattara A, Langeron O, Coriat P, Riou B. Overestimation of Bispectral Index in sedated intensive care unit patients revealed by administration of muscle relaxant. Anesthesiology 2003; 99: 9-17. 